\title{
Long-term effects of maternal fish oil intake in young pigs challenged with lipopolysaccharide
}

\author{
A. S. Souza ${ }^{1}$, J. E. Bolhuis ${ }^{1}$, W. J. J. Gerrits ${ }^{2}$ and A. Lammers ${ }^{1}$ \\ ${ }^{1}$ Adaptation Physiology Group and ${ }^{2}$ Animal Nutrition Group, Department of Animal Science, Wageningen University, \\ The Netherlands
}

Evidence suggests that $\omega 3$ long-chain polyunsaturated fatty acid (LC-PUFA) intake may have benefits on general health ${ }^{(1)}$. Up to date, there has been not much information about the long-term impact of maternal $\omega 3$ LCPUFA intake on the offspring ${ }^{(2)}$. Using swine as model species, we therefore examined the effects of maternal fish oil (FO, rich in the $\omega 3$ LCPUFA DHA) intake on brain fatty acid (FA) profile, and the behavioural, febrile and immunological response of the offspring challenged with lipopolysaccharide (LPS).

We included maternal diet $\left(20 \mathrm{~g} / \mathrm{kg}\right.$ Marinol ${ }^{\mathrm{TM}} \mathrm{D}-40$ containing about $40 \%$ DHA, FO treatment, or $20 \mathrm{~g} / \mathrm{kg}$ high oleic acid sunflower oil containing no $\omega 3 \mathrm{FA}$, HOSF treatment) and offspring LPS injection (LPS or saline) as treatments. Forty-eight pigs (12 pens, 6 pens/ dietary treatment) were subjected to the LPS challenge (Escherichia coli LPS, serotype O111:B4; Sigma Aldrich, NL) at 13 weeks of age, 9 weeks after weaning. They were given a single i.v. injection of saline or LPS $\left(2 \mu \mathrm{g} / \mathrm{kg} \mathrm{BW}^{3}\right)$. In each pen, three pigs were injected with LPS and one with saline. Rectal temperature and blood plasma were sampled during three days. In response to the LPS injection, a decrease in social contact with humans has previously been reported as a sign of increased behavioural sickness response ${ }^{(3)}$. Therefore, voluntary approach towards a test person was used as an indicator of sickness response. For quantification of brain DHA, offspring brains were harvested at 4 and 14 weeks of age.

DHA percentages in frontal cortex and hippocampus presented an age $\times$ maternal diet interaction $(P<0.001)$. Although there was a significant decrease of brain DHA in 14-week-old pigs compared to their 4-week-old siblings, DHA levels were higher in FO than in HOSF pigs at both age groups and brain areas. In the human approach test, a maternal diet $\times$ LPS interaction $(P<0.05)$ was found $1 \mathrm{~h}$ after injection. No effect of LPS on the percentage of FO pigs approaching the person $(P=0.23)$ was found, whereas within the HOSF pigs, fewer LPS-injected than saline-injected pigs approached the person $(P<0.05)$. Three hours after LPS injection, none of HOSF pigs approached the person, whereas about $30 \%$ of FO pigs did approach. Analysis of rectal temperature showed a maternal diet $\times$ LPS $\times$ time interaction $(P<0.001)$. A time-dependent increase in rectal temperature was observed only after LPS injection $(P<0.01)$, with higher temperatures in HOSF pigs $(P<0.01)$ compared to FO ones. High variation in mean values of cytokines was observed. Comparison of baselines and post-injection cytokines showed an overall increase of IFN- $\gamma(P<0.01)$, TNF- $\alpha(P<0.001)$ and IL-6 $(P<0.001)$ after LPS injection. However, the cytokine responses were not affected by the maternal diet. In agreement with other studies, IL-1 $\beta$ was not detected.

In this study, we investigated possible enduring effects of maternal FO intake on the response to an LPS injection in the offspring, as a first step towards untangling the long-term effects of DHA supplementation on animal health. With regard to LPS-induced cytokine levels in plasma, we found no effect of the maternal diet, but differences in sickness behaviour and febrile response were observed. Furthermore, as the differences in brain DHA concentrations between FO and HOSF pigs indicate that maternal intake of FO does result in neural changes in the offspring, further research on the effects of maternal DHA intake on offspring health is warranted.

1. Calder PC (1996) Proc Nutr Soc 55, 737-774.

2. Forsyth JS, Willatts P, Agostoni C et al. (2003) BMJ 326, 953-955.

3. de Groot J, Kranendonk G, Fillerup M et al. (2007) Physiol Behav 90, 612-618. 